\title{
Trace Identities in the Inverse Scattering Transform Method Associated with Matrix Schrödinger Operators
}

ARTICLE in JOURNAL OF MATHEMATICAL PHYSICS · OCTOBER 1982

Impact Factor: 1.18 · DOI: 10.1063/1.525265

CITATIONS

18
DOWNLOADS

81
VIEWS

84

2 AUTHORS:

\section{Luis Martinez Alonso}

Complutense University of Madrid 140 PUBLICATIONS $\quad 1,180$ CITATIONS

SEE PROFILE
Eugenio Olmedilla

Complutense University of Madrid 10 PUBLICATIONS 189 CITATIONS

SEE PROFILE 


\title{
Trace identities in the inverse scattering transform method associated with matrix Schrodinger operators
}

\author{
L. Martinez Alonso and E. Olmedilla \\ Departamento de Métodos Matemáticos de la Física, Facultad de Ciencias Físicas, Universidad Complutense, \\ Madrid-3, Spain
}

(Received 5 February 1982; accepted for publication 14 May 1982)

Trace identities arising in the scattering theory of one-dimensional matrix Schrödinger operators are deduced. They derive from the properties of an asymptotic expansion of the trace of the resolvent kernel in inverse powers of the spectral parameter. Applications of these trace identities for characterizing infinite families of conservation laws for nonlinear evolution equations are given.

PACS numbers: 03.65.Nk, 02.30.Tr

\section{INTRODUCTION}

One of the most important properties of the nonlinear equations which are integrable by means of the inverse scattering transform method is the existence of an infinite family of local conservation laws. A natural explanation of this fact, for the case of the Korteweg-de Vries equation, was provided by Faddeev and Zakharov ${ }^{1}$ by using the trace identities arising in the scattering transform theory for the Schrödinger equation. Shortly thereafter trace identities for other spectral problems were applied to the analysis of important nonlinear wave equations as, for instance, the nonlinear Schrödinger equation ${ }^{2}$ and the sine-Gordon equation. ${ }^{3}$ The interest in trace identities is not only motivated by their connection with conservation laws; they are also a fundamental step in arriving at a description of completely integrable equations in terms of systems of action-angle variables. This latter application of trace identities is particularly relevant in the semiclassical quantization of completely integrable wave equations $\mathrm{s}^{4.5}$ as well as in the quantum inverse scattering transform method. ${ }^{6}$

In this paper we derive the trace identities associated with general matrix Schrödinger spectral problems. The inverse scattering transform theory for Hermitian matrix Schrödinger operators was analyzed by Wadati and Kamijo ${ }^{7}$ who also indicated explicit examples of Lax pair equations. On the basis of this theory, Calogero and Degasperis ${ }^{8}$ deduced a wide class of nonlinear evolution equations for which the scattering data evolve in a simple form. However, as already observed by Wadati and Kamijo, several of the more important evolution equations appear to be related to non-Hermitian matrix Schrödinger operators, for which a general inverse scattering transform theory has not yet been formulated.

The starting point of our derivation of trace identities is the asymptotic expansion of the trace of the resolvent operator. This expansion can be obtained in two different ways: firstly, by using the algebraic properties of the diagonal of the resolvent kernel in the context of symbolic calculus of differential operators," and, secondly, by means of the analytic properties of the trace of the resolvent as a function of the spectral parameter. The first procedure provides an asymptotic expansion in which the coefficients appear as functionals with polynomial densities depending on the matrix elements of the potential and their derivatives. By the second procedure these coefficients can be expressed in terms of scattering data and this leads us to the trace identities. The paper is organized as follows.

Section 2 deals with the main properties of the Jost solutions and the resolvent kernel for matrix Schrödinger operators. Several of these properties, those included in Theorems 1 and 2, are listed without proof since they derive easily from methods which are similar to the ones used in the scalar case. ${ }^{10,11}$ We do, however, provide a detailed proof of Proposition 1 of Sec. 2 since it leads to the characterization of bound states as zeros of the determinant of the Wronskian of two matrix Jost solutions. Section 3 is concerned with the derivation of the trace identities. In Part A a recursion relation is given which enables us to calculate explicitly the coefficients $H_{n}$ of the asymptotic expansion for the trace of the resolvent operator. In Part B of Sec. 3 we use the analyticity properties of the trace of the resolvent in order to get the expressions of the coefficients $H_{n}$ in terms of scattering data. Finally, Sec. 4 includes the application of the trace identities to obtain infinite sets of conservation laws for several relevant families of integrable nonlinear evolution equations.

\section{MATRIX SCHRÖDINGER SPECTRAL PROBLEMS A. Notation and basic properties}

We will be concerned with the $N \times N$ matrix Schrödinger operator

$$
L=-\partial_{x x}+V(x), \quad-\infty<x<\infty,
$$

where $V(x)$ is a complex $N \times N$ matrix function, non-Hermitian in general, satisfying ${ }^{12}$

$$
\int_{-\infty}^{\infty}\left(1+x^{2}\right)|V(x)| d x<\infty .
$$

Here and below we denote $|\boldsymbol{M}|=\max _{i} \boldsymbol{\Sigma}_{k}\left|\boldsymbol{M}_{j k}\right|$ for a given matrix $M$. The operator $L$ acts on vector functions with $N$ components, but in studying its spectral properties it proves useful to consider the following two eigenvalue problems:

$$
\begin{aligned}
& -\partial_{x x} F+V(x) F=k^{2} F, \\
& -\partial_{x x} \bar{F}+\bar{F} V(x)=k^{2} \bar{F},
\end{aligned}
$$

where both $F$ and $\bar{F}$ are assumed to be $N \times N$ matrix functions. We define the Jost solutions $F_{ \pm}$of $(2.3 \mathrm{a})$ and $\bar{F}_{ \pm}$of (2.3b) as those matrix functions verifying the integral equations 
$F_{ \pm}(k, x)=e^{ \pm i k x} 1-\int_{x}^{ \pm \infty} \frac{\sin k(x-y)}{k} V(y) F_{ \pm}(k, y) d y$,

$\bar{F}_{ \pm}(k, x)=e^{ \pm i k x} \mathbb{1}-\int_{x}^{ \pm \infty} \frac{\sin k(x-y)}{k} \bar{F}_{ \pm}(k, y) \boldsymbol{V}(y) d y$,

where $\mathbb{I}$ is the $N \times N$ identity matrix. These integral equations can be analyzed by means of the well-known method of successive approximations. ${ }^{10,11}$ In order to describe the results which are derived from this method we introduce the two subsets

$$
\mathbb{C}^{+}=\{k \in \mathbb{C}: \operatorname{Im} k>0\}, \quad \overline{\mathrm{C}}^{+}=\{k \in \mathbb{C}: \operatorname{Im} k \geqslant 0\} .
$$

One proves the following theorem.

Theorem 1: The Jost solutions $F_{ \pm}(k, x), \bar{F}_{ \pm}(k, x)$ exist for all $k \in \overline{\mathbb{C}}^{+}$and, as functions of $k$, are analytic on $\mathbb{C}^{+}$and continuous on $\overline{\mathbb{C}}^{+}$. Moreover, they satisfy the bounds ${ }^{13}$

$$
\left|e^{\mp i k x} \widetilde{F}_{ \pm}(k, x)\right|=1+O(1 /|k|), \quad|k| \rightarrow \infty,
$$

where $\widetilde{F}_{ \pm}$denotes either $F_{ \pm}$or $\widetilde{F}_{ \pm}$.

Given two solutions $F$ and $\bar{F}$ of $(2.3 \mathrm{a})$ and (2.3b), respectively, the Wronskian

$$
[\bar{F} ; F]=\bar{F}\left(\partial_{x} F\right)-\left(\partial_{x} \bar{F}\right) F
$$

is independent of $x$. This property leads at once to the following relations, valid for all nonzero real $k$ :

$$
\begin{aligned}
& F_{+}(k, x)=F_{-}(-k, x) A(k)+F_{-}(k, x) B(k), \\
& F_{-}(k, x)=F_{+}(-k, x) C(k)+F_{+}(k, x) D(k), \\
& \bar{F}_{+}(k, x)=C(k) \bar{F}_{-}(-k, x)-D(-k) \bar{F}_{-}(k, x), \\
& \bar{F}_{-}(k, x)=A(k) \bar{F}_{+}(-k, x)-B(-k) \bar{F}_{+}(k, x),
\end{aligned}
$$

where $A, B, C, D$ are the matrices defined by

$$
\begin{aligned}
& A(k)=(1 / 2 i k)\left[\bar{F}_{-}(k) ; F_{+}(k)\right], \\
& C(k)=-(1 / 2 i k)\left[\bar{F}_{+}(k) ; F_{-}(k)\right], \\
& B(k)=-(1 / 2 i k)\left[\bar{F}_{-}(-k) ; F_{+}(k)\right], \\
& D(k)=(1 / 2 i k)\left[\bar{F}_{+}(-k), F_{-}(k)\right] .
\end{aligned}
$$

The compatibility of the relations (2.8)-(2.11) implies the constraints

$$
\begin{aligned}
& C(-k) A(k)+D(k) B(k) \\
& \quad=A(-k) C(k)+B(k) D(k)=1, \\
& D(-k) A(k)+C(k) B(k) \\
& =B(-k) C(k)+A(k) D(k)=0 .
\end{aligned}
$$

The matrices $A(k)$ and $C(k)$ are specially important for our subsequent discussion on the trace identities. Their definition as given in (2.12) can be extended to $\operatorname{Im} k>0$, and the properties of the Jost solutions together with the analysis of the integral equations (2.4) imply

Theorem 2: The matrix functions $A(k)$ and $C(k)$ are analytic on $\mathbb{C}^{+}$and continuous on $\overline{\mathrm{C}}^{+}-\{0\}$. Moreover, they satisfy

$$
\begin{aligned}
& |A(k)-\mathbb{1}|=O(1 /|k|), \quad|C(k)-\mathbb{1}|=O(1 /|k|), \\
& |k| \rightarrow \infty .
\end{aligned}
$$

As we shall see later, $\operatorname{det} A(k)=\operatorname{det} C(k)$, but at this point of our exposition it is convenient to content ourselves with the following partial result.

Proposition 1: The functions $\operatorname{det} A(k)$ and $\operatorname{det} C(k)$ have the same set of zeros in $\overline{\mathbb{C}}^{+}-\{0\}$.

Proof: Suppose we have $k_{0} \in \overline{\mathbb{C}}^{+}-\{0\}$ such that $\operatorname{det} A\left(k_{0}\right)=0$. Then there will be a nonzero vector such that $A\left(k_{0}\right) a=0$. According to $(2.12)$ this implies that

$$
\bar{F}_{-}\left(k_{0}, x\right) \partial_{x} \varphi(x)=\left(\partial_{x} \bar{F}_{-}\left(k_{0}, x\right)\right) \varphi(x),
$$

where $\varphi(x) \equiv F_{+}\left(k_{0}, x\right) a$. Due to the asymptotic behavior of $\bar{F}_{-}$as $x \rightarrow-\infty$, the matrix $\bar{F}_{-}\left(k_{0}, x\right)$ will be invertible for all $x$ in some interval $(-\infty, r)$. Therefore $(2.16)$, considered as a first-order differential equation for $\varphi(x)$, has $N$ linearly independent solutions defined on $(-\infty, r)$. But since the Wronskian $\left[\bar{F}_{-}\left(k_{0}\right) ; F_{-}\left(k_{0}\right)\right]$ vanishes, each column of $F_{-}\left(k_{0}, x\right)$ is a solution of (2.16). In addition, these columns are linearly independent for all $x$ in some interval $\left(-\infty, r^{\prime}\right)$. Hence, for all $x$ in the interval $\left(-\infty, \min \left(r, r^{\prime}\right)\right)$ the general solution of (2.16) is a linear combination of the columns of $F_{-}\left(k_{0}, x\right)$. As a consequence a nonzero vector $b$ exists verifying

$$
F_{+}\left(k_{0}, x\right) a=F_{-}\left(k_{0}, x\right) b .
$$

Note that this equation must hold for all $x \in \mathbb{R}$ since $F_{+}\left(k_{0}, x\right) a$ and $F_{-}\left(k_{0}, x\right) b$ are solutions of $\left(L-k^{2}\right) \varphi=0$, and then, if they coincide on an interval they coincide also on the whole line $-\infty<x<\infty$. Now, if we use the definition of $C(k)$ as given in (2.12) we have

$$
C\left(k_{0}\right) b=-\left(1 / 2 i k_{0}\right)\left[\bar{F}_{+}\left(k_{0}\right) ; F_{+}\left(k_{0}\right)\right] a=0 .
$$

That is to say, det $C\left(k_{0}\right)=0$. In a similar fashion, one proves that each zero of $\operatorname{det} C(k)$ is also a zero of $\operatorname{det} A(k)$. Q.E.D. Henceforth we will denote

$$
Z=\left\{k \in \overline{\mathrm{C}}^{+}-\{0\}: \operatorname{det} \boldsymbol{A}(k)=\operatorname{det} C(k)=0\right\} .
$$

Obviously, from (2.17) it follows that each $k \in Z \cap \mathbb{C}^{+}$determines an exponentially decreasing eigenfunction $F_{+}(k, x) a=F_{-}(k, x) b$ of the matrix Schrödinger operator $L$, and then $k^{2}$ is a proper eigenvalue of $L$. However, we notice that if the potential matrix $V(x)$ is non-Hermitian then the set $Z$ may have elements with $\operatorname{Im} k=0$ and these do not correspond to bound states of $L$.

From the point of view of scattering theory, the Jost solutions $F_{+}$and $F_{-}$describe waves incident from the left and the right, respectively. In this way, taking into account the relations (2.8) and (2.9) we deduce that the transmission and reflection coefficients are given by

$$
T(k)=C(k)^{-1}, \quad R(k)=D(k) C(k)^{-1},
$$

in the case of right incidence, and by

$$
\hat{T}(k)=A(k)^{-1}, \quad \hat{R}(k)=B(k) A(k)^{-1},
$$

for the left-incidence case. Moreover, by Eqs. (2.14) one readily finds the following relations:

$$
\begin{aligned}
& T(-k)=(\mathbb{1}-\hat{R}(-k) \hat{R}(k)) \hat{T}(k)^{-1}, \\
& R(k)=-\widehat{T}(k) \hat{R}(-k) \widehat{T}(-k)^{-1} .
\end{aligned}
$$

\section{B. The resolvent kernel}

The resolvent operator of $L$ is an integral operator acting on $N$-component functions and its kernel admits the following representation in terms of the Jost solutions of (2.3a) and $(2.3 \mathrm{~b})$ : 


$$
R(k, x, y)= \begin{cases}(i / 2 k) F_{+}(k, x) A(k)^{-1} \bar{F}_{-}(k, y), & x \geqslant y, \\ (i / 2 k) F_{-}(k, x) C(k)^{-1} \bar{F}_{+}(k, y) . & x \leqslant y\end{cases}
$$

In order to justify this fact let us prove

Lemma 1: Given $k \in \overline{\mathbb{C}}^{+}-(Z \cup\{0\})$ and $x_{0} \in \mathbb{R}$, then the matrix functions

$$
\begin{aligned}
F(x)= & (1 / 2 i k)\left(F_{+}(k, x) A(k)^{-1} \bar{F}_{-}\left(k, x_{0}\right)\right. \\
& \left.-F_{-}(k, x) C(k)^{-1} \bar{F}_{+}\left(k, x_{0}\right)\right)
\end{aligned}
$$

and

$$
\begin{aligned}
\bar{F}(x)= & (i / 2 k)\left(F_{+}\left(k, x_{0}\right) A(k)^{-1} \bar{F}_{-}(k, x)\right. \\
& \left.-F_{-}\left(k, x_{0}\right) C(k)^{-1} \bar{F}_{+}(k, x)\right)
\end{aligned}
$$

are solutions of (2.3a) and (2.3b), respectively, verifying

$$
F\left(x_{0}\right)=\bar{F}\left(x_{0}\right)=0, \quad\left(\partial_{x} F\right)\left(x_{0}\right)=\left(\partial_{x} \bar{F}\right)\left(x_{0}\right)=\mathbb{1} .
$$

Proof: Consider the function $F(x)$; clearly it is a solution of (2.3a) and it verifies the Wronskian relations

$$
\begin{aligned}
& {\left[\bar{F}_{+}(k) ; F\right]=\bar{F}_{+}\left(k, x_{0}\right),} \\
& {\left[\bar{F}_{-}(k) ; F\right]=\bar{F}_{-}\left(k, x_{0}\right) .}
\end{aligned}
$$

If we think of (2.26a) as a first-order differential equation for $F$ it is easy to conclude that $F$ must be of the form

$$
F(x)=F_{+}(k, x) M+F_{0}(k, x),
$$

where $M$ is some constant matrix and $F_{0}$ stands for the solution of (2.3a) satisfying the boundary conditions (2.25). Now, if $(2.27)$ is inserted into $(2.26 \mathrm{~b})$ and the Wronskian $\left[\bar{F}_{-} ; F\right]$ is evaluated at $x=x_{0}$, it follows at once that $M=0$. Therefore $F=F_{0}$ and the enunciated property for $F$ is proved. The proof of the statement for $\bar{F}$ is completely similar. Q.E.D.

As a consequence of this lemma, it follows that $R(k, x, y)$ verifies the equations

$$
\begin{aligned}
& -\partial_{x x} R(k, x, y)+\left(V(x)-k^{2}\right) R(k, x, y)=\delta(x-y), \\
& -\partial_{x x} R(k, y, x)+R(k, y, x)\left(V(x)-k^{2}\right)=\delta(x-y) .
\end{aligned}
$$

Now, from the properties of the Jost solutions it can be seen that for $k \in \mathbb{C}^{+}-Z$ the function $R(k, x, y)$ decreases exponentially as $(x, y)$ goes to infinity. Hence (2.28) and (2.29) mean that the integral operator determined by the kernel $R(k, x, y)$ is a bounded two-sided inverse operator of $\left(L-k^{2}\right)$ provided that $k \in \mathbb{C}^{+}-Z$. Furthermore, it is clear that, as a function of $k, R(k, x, y)$ is analytic on $\mathbb{C}^{+}-Z$ and continuous on $\overline{\mathrm{C}}^{+}-(Z \cup\{0\})$. All these properties imply at once that $R(k, x, y)$ is the kernel of the resolvent operator of $L$.

In a recent paper ${ }^{14}$ Ragnisco has given an expression for the kernel of the resolvent operator which contains some trivial misprints: the resolvent kernel $R^{(-)}(x, y, k)$ should change sign, and it is analytically continuable in the upper (not lower) half $k$-plane.

\section{DERIVATION OF THE TRACE IDENTITIES A. The trace of the resolvent operator}

As it has been shown by Gel'fand and Dikii, ${ }^{9}$ the diagonal of the resolvent kernel of a differential operator is an interesting algebraic object. In the case of the matrix Schrödinger operator (2.1) we have that according to $(2.23)$ the restriction of $R(k, x, y)$ to the diagonal $x=y$ is given by the matrix function

$$
\begin{aligned}
R(k, x) & =(i / 2 k) F_{+}(k, x) A(k)^{-1} \bar{F}_{-}(k, x) \\
& =(i / 2 k) F_{-}(k, x) C(k)^{-1} \bar{F}_{+}(k, x) .
\end{aligned}
$$

Observe that the equality between both expressions for $R(k, x)$ is a consequence of Lemma 1. Following the method of Gel'fand-Diki based on the symbolic calculus of differential operators one proves ${ }^{9,15}$ the existence of an asymptotic expansion of $R(k, x)$ for $|k| \rightarrow \infty$;

$$
R(k, x)=\frac{i}{2 k} \sum_{n=0}^{\infty} R_{n}(x) k^{-2 n}, \quad R_{0}=1,
$$

where the coefficients $R_{n}(n \geqslant 1)$ are polynomials, without constant term, depending on the potential $V(x)$ and its derivatives. The explicit form of these coefficients can be calculated by taking into account the expression (3.1) for $R(k, x)$ as a product of solutions of (2.3a) and (2.3b). Indeed, one readily finds that (2.3a) and (2.3b) imply

$$
L_{V} R(k, x)=k^{2} \partial_{x} R(k, x)
$$

where $L_{V}$ is the operator defined by

$$
\begin{aligned}
L_{V} R \equiv & \frac{1}{4}\left(-\partial_{x x x} R+\partial_{x}\{V, R\}+\left\{V, \partial_{x} R\right\}\right. \\
& \left.-\left[V, \int_{+\infty}^{x}[V, R]\left(x^{\prime}\right) d x^{\prime}\right]\right),
\end{aligned}
$$

and $\{$,$\} denotes the anticommutator operation for matri-$ ces. Insertion of (3.2) into (3.3) leads to the recursion relation

$$
\partial_{x} R_{n+1}=L_{V} R_{n}, \quad R_{0}=1,
$$

which allows us to calculate the coefficients $R_{n}$. For example, one obtains

$$
\begin{aligned}
& R_{1}=\frac{1}{2} V, \quad R_{2}=-\frac{1}{8}\left(V_{x x}-3 V^{2}\right), \\
& R_{3}=\frac{1}{32}\left(V_{x x x}-5\left(V^{2}\right)_{x x}+5 V_{x}^{2}+10 V^{3}\right) .
\end{aligned}
$$

We define the trace of the resolvent operator of $L$ as the following integral:

$$
\int_{-\infty}^{\infty} \operatorname{tr}\left(R(k, x)-\frac{i}{2 k} \mathbb{1}\right) d x
$$

where $\operatorname{tr}()$ denotes the trace operation of matrices. The analysis of the integral equations (2.4) shows that for $\operatorname{Im} k>0$ the Jost solutions verify the asymptotic behavior ${ }^{16}$

$$
\begin{aligned}
& F_{+}(k, x) \underset{x \rightarrow-\infty}{\sim} A(k) e^{i k x}, F_{-}(k, x) \underset{x \rightarrow+\infty}{\sim} C(k) e^{-i k x}, \\
& \bar{F}_{+}(k, x) \underset{x \rightarrow-\infty}{\sim} C(k) e^{i k x}, \bar{F}_{-}(k, x) \underset{x \rightarrow+\infty}{\sim} A(k) e^{-i k x} .
\end{aligned}
$$

Then by (3.1) we have that the integral (3.7) converges for $k \in \mathbb{C}^{+}-Z$. Now, substitution of (3.2) into (3.7) yields the asymptotic expansion

$$
\int_{-\infty}^{\infty} \operatorname{tr}\left(R(k, x)-\frac{i}{2 k} \mathbb{1}\right) d x=\frac{i}{2 k} \sum_{n=1}^{\infty} H_{n}[V] k^{-2 n},
$$

where the coefficients $H_{n}[V]$ are the functionals depending on $V$ given by 


$$
H_{n}[V]=\int_{-\infty}^{\infty} \operatorname{tr} R_{n}(x) d x
$$

The above mentioned properties of the coefficients $R_{n}$ imply that the densities of the functionals $H_{n}$ are polynomials depending on the matrix elements of the potential $V$ and their derivatives with respect to $x$. For instance, the first few functionals are

$$
\begin{gathered}
H_{1}[V]=\frac{1}{2} \int_{-\infty}^{\infty} \operatorname{tr} V d x, \quad H_{2}[V]=\frac{3}{8} \int_{-\infty}^{\infty} \operatorname{tr} V^{2} d x \\
H_{3}[V]=\frac{5}{32} \int_{-\infty}^{\infty} \operatorname{tr}\left(V_{x}^{2}+2 V^{3}\right) d x
\end{gathered}
$$

\section{B. Trace identities}

We are going to obtain another expression for the asymptotic expansion (3.9). Our derivation is based on the following identity:

Proposition 2: For all $k \in \mathbb{C}^{+}-Z$ it is verified that

$$
\begin{aligned}
\int_{-\infty}^{\infty} \operatorname{tr}\left(R(k, x)-\frac{i}{2 k} \mathbb{1}\right) d x & =-\frac{1}{2 k} \frac{\partial_{k} \operatorname{det} A(k)}{\operatorname{det} A(k)} \\
& =-\frac{1}{2 k} \frac{\partial_{k} \operatorname{det} C(k)}{\operatorname{det} C(k)} .
\end{aligned}
$$

Proof: Given two solutions $F$ and $\bar{F}$ of (2.3a) and (2.3b), respectively, we have the identity

$$
\partial_{x}\left[\bar{F}(k) ; \partial_{k} F(k)\right]=-2 k \bar{F}(k) F(k) .
$$

Thus, from (3.1) and (3.13) we get

$$
\begin{aligned}
\operatorname{tr} R(k, x) & =-\left(i / 4 k^{2}\right) \partial_{x} \operatorname{tr}\left[A(k)^{-1} \bar{F}_{-}(k, x) ; \partial_{k} F_{+}(k, x)\right] \\
& =-\left(i / 4 k^{2}\right) \partial_{x} \operatorname{tr}\left[C(k)^{-1} \bar{F}_{+}(k, x) ; \partial_{k} F_{-}(k, x)\right] .
\end{aligned}
$$

In this way, taking into account the asymptotic behavior (3.8) of the Jost solutions for $\operatorname{Im} k>0$, one finds

$$
\begin{aligned}
\int_{-\infty}^{\infty} \operatorname{tr}\left(R(k, x)-\frac{i}{2 k} \mathbb{1}\right) d x \\
=-\frac{1}{2 k} \operatorname{tr}\left(A(k)^{-1} \partial_{k} A(k)\right) \\
=-\frac{1}{2 k} \operatorname{tr}\left(C(k)^{-1} \partial_{k} C(k)\right) .
\end{aligned}
$$

Therefore, by using the matrix identity

$$
\partial_{k} \operatorname{det} M(k)=\operatorname{det} M(k) \operatorname{tr}\left(M(k)^{-1} \partial_{k} M(k)\right),
$$

the result follows.

Now, we are ready to improve the statement given in Proposition 1.

Proposition 3: $\operatorname{det} A(k)=\operatorname{det} C(k)$ for all $k \in \overline{\mathrm{C}}^{+}-\{0\}$.

Proof: From (3.12) we have that

$$
\partial_{k}\left(\frac{\operatorname{det} A(k)}{\operatorname{det} C(k)}\right)=0 \text {, }
$$

for all $k \in \mathbb{C}^{+}-Z$. Because of the analyticity of $\operatorname{det} A(k)$ on the open set $\mathbb{C}^{+}$the zeros of $\operatorname{det} A(k)$ on $\mathbb{C}^{+}$are isolated points and then $\mathrm{C}^{+}-Z$ is an open connected set. Hence, by (3.17) and due to the fact that

$$
\lim _{|k| \rightarrow \infty} \frac{\operatorname{det} A(k)}{\operatorname{det} C(k)}=1,
$$

we deduce that $\operatorname{det} A(k)$ and $\operatorname{det} C(k)$ coincide on $\mathbb{C}^{+}-Z$. Moreover, since both functions are continuous on $\overline{\mathbb{C}}^{+}-\{0\}$, they coincide also on $\overline{\mathbb{C}}^{+}-\{0\}$.

Q.E.D.

Because det $A(k) \rightarrow 1$ as $|k| \rightarrow \infty$ there will be some $r>0$ such that det $A(k)$ is never 0 on the simple connected open set $\mathbb{C}_{r}^{+} \equiv\left\{k \in \mathbb{C}^{+}:|k|>r\right\}$. Consequently, det $A(k)$ hasan analytic logarithm on $\mathbb{C}_{r}^{+}$and Eq. (3.12) may be rewritten as

$$
\begin{aligned}
\int_{-\infty}^{\infty} \operatorname{tr} & \left(R(k, x)-\frac{i}{2 k} \mathbb{1}\right) d x \\
& =-\frac{1}{2 k} \partial_{k} \ln [\operatorname{det} A(k)], \quad k \in \mathbb{C}_{r}^{+} .
\end{aligned}
$$

At this point we make the assumption that no zero of $\operatorname{det} A(k)$ lies on the real axis. This is satisfied automatically when the potential $V(x)$ is Hermitian, but it is not assured in the general case. From this assumption and the properties of $\operatorname{det} A(k)$ we deduce that $Z$ is a finite set

$\left\{k_{1}: 1=1, \ldots, m\right\} \subset \mathrm{C}^{+}$. Moreover, if we denote by $n_{1}$ the order of the zero $k_{1}(1=1, \ldots, m)$, it follows that the function

$$
f(k) \equiv[\operatorname{det} A(k)] \prod_{l}\left(\frac{k+k_{l}}{k-k_{l}}\right)^{n_{l}}
$$

is analytic on $\mathbb{C}^{+}$, continuous and never 0 on $\overline{\mathrm{C}}^{+}-\{0\}$, and $f(k) \rightarrow 1$ as $|k| \rightarrow \infty$. Hence, $f(k)$ admits a logarithm branch which is analytic on $\mathbb{C}^{+}$, continuous on $\overline{\mathbb{C}}^{+}-\{0\}$, and such that $\ln f(k) \rightarrow 0$ as $|k| \rightarrow \infty$. In this way, according to the Beckenbach's generalization of Cauchy's Integral theorem, ${ }^{17}$ we have

$$
\ln f(k)=\frac{1}{2 \pi i} \oint_{\gamma} \frac{\ln f(z)}{z-k} d z, \quad \operatorname{Im} k>0,
$$

where $\gamma$ is the contour consisting of a semicircle of radius $r$ in the upper half-plane, plus the real axis from $-r$ to $+r$, except the origin which is avoided along a semicircle of radius $\epsilon$. Now, because $\ln f(z) \rightarrow 0$ as $|z| \rightarrow \infty$ the large semicircle does not contribute to the integral (3.21) in the limit $r \rightarrow \infty$. On the other hand, it can be proved ${ }^{18}$ that $|A(k)| \leqslant$ const $/|k|$ as $k \rightarrow 0$. Then $f(k)$ can become infinite when $k \rightarrow 0$ no faster than $k^{-N}$, and it implies that the contribution of the small semicircle to the integral (3.21) vanishes as $\epsilon \rightarrow 0$. Therefore it follows that

$$
\ln f(k)=\frac{1}{2 \pi i} \int_{-\infty}^{\infty} \frac{\ln f(q)}{q-k} d q, \quad \operatorname{Im} k>0 .
$$

Here $f$ signifies that the Cauchy principal value of the integral must be taken at $q=0$. Analogously, by integrating along a similar contour in the lower half-plane, we find

$$
0=\frac{1}{2 \pi i} f_{-\infty}^{\infty} \frac{\ln f(-q)}{q-k} d q, \quad \operatorname{Im} k>0 .
$$

Now, by adding (3.22) and (3.23),

$$
\ln f(k)=\frac{1}{2 \pi i} f_{-\infty}^{\infty} \frac{\ln (f(q) f(-q))}{q-k} d q, \quad \operatorname{Im} k>0 .
$$

Then, by using (3.20), we deduce that for $k \in \mathbb{C}_{r}^{+}$,

$$
\begin{aligned}
\ln [\operatorname{det} A(k)]= & -\sum_{l} \ln \left(\frac{k+k_{l}}{k-k_{l}}\right)^{n_{l}} \\
& +\frac{1}{2 \pi i} \int_{-\infty}^{\infty} \frac{\ln [\operatorname{det}(A(q) A(-q))]}{q-k} d q .(3 .
\end{aligned}
$$


This expression leads to the asymptotic expansion

$$
\begin{aligned}
& \ln [\operatorname{det} A(k)]=\frac{i}{2 k} \sum_{n=0}^{\infty}\left\{\frac{4 i}{2 n+1} \sum_{l} n_{l} k_{l}^{2 n+1}\right. \\
& \left.+\frac{1}{\pi} f_{-\infty}^{\infty} q^{2 n} \ln [\operatorname{det}(A(q) A(-q))] d q\right\} k^{-2 n} .
\end{aligned}
$$

In addition, from Proposition 3 and the relations (2.22) we have

$$
\operatorname{det}(A(q) A(-q))=[\operatorname{det}(\mathbb{1}-R(q) R(-q))]^{-1} .
$$

Therefore, by substituting (3.9) and (3.26) into (3.19), we get that the coefficients of the asymptotic expansion of the trace of the resolvent operator may be expressed in terms of scattering data in the following form:

$$
\begin{aligned}
& H_{n}[V]=2 i \sum_{l} n_{l} k_{l}^{2 n-1}-\frac{2 n-1}{2 \pi} \\
& \quad \times \int_{-\infty}^{\infty} q^{2 n-2} \ln [\operatorname{det}(\mathbb{1}-R(q) R(-q))] d q, \quad n \geqslant 1 .
\end{aligned}
$$

These relations are the trace identities for matrix Schrödinger operators. Observe that the existence of the integrals appearing in (3.28) requires that $R(q)$ must decrease rapidly as $|q| \rightarrow \infty$. This asymptotic behavior for $R(q)$ is assured by assuming that the potential $V(x)$ is infinitely differentiable and along with its derivatives decreases rapidly at infinity. ${ }^{19}$

\section{CONSERVATION LAWS FOR NONLINEAR EVOLUTION EQUATIONS}

By means of the trace identities (3.30) we can derive in a unified way infinite families of conservation laws for several of the most important nonlinear evolution equations solvable by the inverse scattering transform method. To see this, let us consider the Calogero-Degasperis equations ${ }^{8}$ for $N \times N$ matrix functions $V(x, t)$;

$$
\partial_{t} V=2 \beta_{0}(\underline{L}, t) V_{x}+\alpha_{n}(\underline{L})\left[\sigma_{n}, V\right]+\beta_{n}(\underline{L}) \underline{G} \sigma_{n},
$$

where the notation conventions are as in Ref. 8. It was proved by Calogero and Degasperis that the evolution law of the scattering data of the matrix Schrödinger equation under the flows $(4.1)$ is such that the eigenvalues remain invariant and the reflection coefficient for right incidence evolves in time according to

$$
\begin{aligned}
R(k, t)= & \exp \left[4 i k \int_{0}^{t} d t^{\prime} \beta_{0}\left(-4 k^{2}, t^{\prime}\right)\right] \\
& \times \exp \left[t\left(\alpha_{n}\left(-4 k^{2}\right)+2 i k \beta_{n}\left(-4 k^{2}\right)\right) \sigma_{n}\right] \\
& \times R(k, 0) \exp \left[t \left(-\alpha_{n}\left(-4 k^{2}\right)\right.\right. \\
& \left.\left.+2 i k \beta_{n}\left(-4 k^{2}\right)\right) \sigma_{n}\right] .
\end{aligned}
$$

Thus, we find that

$$
\begin{aligned}
R(k, t) R(-k, t)= & \exp \left[t\left(\alpha_{n}\left(-4 k^{2}\right)+2 i k \beta_{n}\left(-4 k^{2}\right)\right) \sigma_{n}\right] \\
& \times R(k, 0) R(-k, 0) \exp \left[-t\left(\alpha_{n}\left(-4 k^{2}\right)\right.\right. \\
& \left.\left.+2 i k \beta_{n}\left(-4 k^{2}\right)\right) \sigma_{n}\right]
\end{aligned}
$$

and then we deduce

$$
\begin{aligned}
& \operatorname{det}(\mathbb{1}-R(k, t) R(-k, t)) \\
& \quad=\operatorname{det}(\mathbb{1}-R(k, 0) R(-k, 0)) .
\end{aligned}
$$

Therefore, from the trace identities (3.28) it is obvious that the functionals $H_{n}[V]$ are conserved under the evolution equations (4.1). We emphasize that the equations (4.1), for which our result applies, contain the time-dependent term $2 \beta_{0}(\underline{L}, t) V_{x}$.

The class of evolution equations (4.1) includes, ${ }^{20}$ among others, the $\mathrm{KdV}$ equation, the modified $\mathrm{KdV}$ equation, the nonlinear Schrödinger equation, and the sine-Gordon equation. In this way, from the functionals $H_{n}[V]$ we can get conservation laws for such equations by means of appropriate choices of the matrix function $V$. For example

(1) Modified Korteweg-de Vries equation:

$$
q_{t}=q_{x x x}+6 q^{2} q_{x}, \quad V=\left(\begin{array}{cc}
-q^{2} & q_{x} \\
-q_{x} & -q^{2}
\end{array}\right) .
$$

(2) Nonlinear Schrödinger equation:

$$
i \psi_{t}=-\psi_{x x}-2|\psi|^{2} \psi, \quad V=\left(\begin{array}{cc}
-|\psi|^{2} & \psi_{x} \\
-\psi_{x}^{*} & -|\psi|^{2}
\end{array}\right)
$$

(3) Sine-Gordon equation:

$$
u_{t x}=\sin u, \quad V=\left(\begin{array}{cc}
-\frac{1}{4} u_{x}^{2} & -\frac{1}{2} u_{x x} \\
\frac{1}{2} u_{x x} & -\frac{1}{4} u_{x}^{2}
\end{array}\right) .
$$

In order to understand the relationship among these sets of conservations laws and the ones already known for these equations, let us consider the generalized ZakharovShabat spectral problem

$$
\left(\partial_{x}-Q+i k \sigma_{3}\right) \varphi=0, \quad Q(x)=\left(\begin{array}{cc}
0 & q(x) \\
r(x) & 0
\end{array}\right),
$$

and its Jost solutions $\psi(k, x)$ and $\bar{\psi}(k, x)$, verifying the conditions

$$
\begin{aligned}
& \left(\begin{array}{l}
\bar{b}(k) e^{-i k x} \\
a(k) e^{i k x}
\end{array}\right) \underset{x \rightarrow-\infty}{\leftarrow} \psi(k, x) \underset{x \rightarrow+\infty}{\rightarrow}\left(\begin{array}{l}
0 \\
1
\end{array}\right) e^{i k x} \\
& \left(\begin{array}{c}
\bar{a}(k) e^{-i k x} \\
-b(k) e^{i k x}
\end{array}\right) \underset{x \rightarrow-\infty}{\leftarrow} \bar{\psi}(k, x) \underset{x \rightarrow+\infty}{\rightarrow}\left(\begin{array}{l}
1 \\
0
\end{array}\right) e^{-i k x}
\end{aligned}
$$

Now, by defining $V=Q^{2}+Q_{x}$ it follows that

$$
-\partial_{x x}+V-k^{2}=\left(-\partial_{x}-Q+i k \sigma_{3}\right)\left(\partial_{x}-Q+i k \sigma_{3}\right)
$$

This implies that the Jost function $F_{+}(k, x)$ of the $2 \times 2$ matrix Schrödinger problem with potential $V=Q^{2}+Q_{x}$ is given by

$$
F_{+}(k, x)=(\bar{\psi}(-k, x) \psi(k, x)) ;
$$

hence, the corresponding matrix $A(k)$ is

$$
A(k)=\left(\begin{array}{cc}
\bar{a}(-k) & 0 \\
0 & a(k)
\end{array}\right) .
$$

Therefore, by (3.9), (3.19), and (4.13) it follows that

$$
\begin{aligned}
& \frac{i}{2 k} \sum_{n=1}^{\infty} H_{n}\left[Q^{2}+Q_{x}\right] k^{-2 n} \\
& \quad=-\frac{1}{2 k} \partial_{k}[\ln a(k)+\ln \bar{a}(-k)] .
\end{aligned}
$$

On the other hand, it is known ${ }^{21}$ that the conservation laws for the evolution equations solvable by the inverse scattering transform associated with $(4.8)$ are the coefficients of two 
asymptotic expansions for the logarithms of $a(k)$ and $\bar{a}(k)$ of the form

$$
\begin{aligned}
& \ln a(k)=-\sum_{n=1}^{\infty} C_{n}[Q] k^{-n}, \quad \operatorname{Im} k>0, \\
& \ln \bar{a}(k)=\sum_{n=1}^{\infty} C_{n}[Q] k^{-n}, \quad \operatorname{Im} k<0 .
\end{aligned}
$$

As a result we find

$$
H_{n}\left[Q^{2}+Q_{x}\right]=2 i(2 n-1) C_{2 n-1}[Q] .
$$

That is to say, the conservation laws $H_{n}\left[Q^{2}+Q_{x}\right]$ provided by the trace relations (3.28) are members of the known families of conservation laws for the evolution equations associated with the generalized Zakharov-Shabat spectral problems.

Another interesting nonlinear equation for which the functionals $H_{n}[V]$ determine an infinite set of conservation laws is the $m$-component nonlinear Schrödinger equation

$$
i \partial_{t} \psi_{\alpha}=-\partial_{x x} \psi_{\alpha}-2 \sum_{\beta=1}^{m}\left|\psi_{\beta}\right|^{2} \psi_{\alpha}, \quad \alpha=1, \ldots, m .
$$

As it has been observed by Wadati, ${ }^{22}$ if we define the $(m+1) \times(m+1)$ matrices

$$
J=\left(\begin{array}{cc}
1 & 0 \cdots 0 \\
0 & \\
\vdots & -\mathbb{1}_{m \times m} \\
0 &
\end{array}\right), \quad U(x)=\left(\begin{array}{cc}
0 & \psi_{1}(x) \cdots \psi_{m}(x) \\
\psi_{1}^{*}(x) & \\
\vdots & \mathbb{O}_{m \times m} \\
\psi_{m}^{*}(x) &
\end{array}\right) \text {, }
$$

then, under the evolution law associated with (4.17), the matrix Schrödinger operator

$$
L=-\partial_{x x}+V(x), \quad V \equiv-U^{2}+J U_{x}
$$

evolves according to the Lax pair equation

$$
\partial_{t} L=[B, L], \quad B \equiv 2 i J \partial_{x x}-2 i U \partial_{x}-i J V .
$$

From (4.20) it is clear that the eigenvalues of $L$ are constants of the motion. Furthermore, it is easy to realize that the reflection coefficient for right incidence associated with $L$ evolves in time in the following form:

$$
R(k, t)=\exp \left(-2 i k^{2} t J\right) R(k, 0) \exp \left(2 i k^{2} t J\right) .
$$

This implies that $\operatorname{det}(\mathbf{1}-R(k, t) R(-k, t))$ is time independent. Therefore, by virtue of the trace identities (3.28), we have that the functionals $H_{n}\left[-U^{2}+J U_{x}\right]$ are conservation laws for the evolution equation (4.17).

'L. D. Faddeev and V. E. Zakharov, Funkcional Anal. Priložen. 5, 18 (1971).

${ }^{2}$ V. E. Zakharov and S. V. Manakov, Theor. Math. Phys. 19, 332 (1974).

${ }^{3}$ L. D. Faddeev and L. A. Takhtajan, Theor. Math. Phys. 21, 160 (1974).

${ }^{4}$ L. D. Faddeev and V. E. Korepin, Phys. Rep. 42, 1 (1978).

${ }^{5}$ L. D. Faddeev, "A Hamiltonian Interpretation of the Inverse Scattering Method" in Solitons, edited by R. K. Bullough and P. J. Caudrey (Springer, Berlin, 1980).

'See the references quoted in the article by M. Fowler, "The Quantum Inverse Scattering Method and Applications to Spin Chains" in Physics in One Dimension, edited by J. Bernasconi and T. Schneider, Springer Series in Solid-State Sciences, Vol. 23 (Springer, Berlin, 1981).

${ }^{7}$ M. Wadati and T. Kamijo, Prog. Theor. Phys. 52, 397 (1974).

${ }^{8}$ F. Calogero and A. Degasperis, Nuovo Cimento B 39, 1 (1977).

'1. M. Gel'fand and L. A. Diki, Usp. Math. Nauk 30, 67 (1975); Funkcional Anal. Priložen. 10, 18 (1976); Funkcional Anal. Priložen. 10, 13 (1976); Funkcional Anal. Priložen. 11, 11 (1977); Funkcional Anal. Priložen. 12, 8 (1978).

${ }^{10}$ L. D. Faddeev, Trudy Mat. Inst. Steklov 73, 314 (1964).

${ }^{11}$ P. Deift and E. Trubowitz, Comm. Pure Appl. Math. 32, 121 (1979).

${ }^{12}$ This condition is more suitable than the usual one, $(1+|x|)|V| \in L^{1}(\mathbf{R})$, in order to characterize the behavior of the scattering coefficients at $k=0$. See Ref. 11

${ }^{13}$ The bounds for $F_{+}$and $\widetilde{F}_{+}$(respectively, $F_{-}$and $\widetilde{F}_{-}$) are uniform with respect to $x$ in the intervals $(\alpha, \infty)$ [respectively, $(-\infty, \beta)$ ], where $\alpha, \beta$ are arbitrary finite numbers.

${ }^{14}$ O. Ragnisco, Lett. Nuovo Cimento 31, 651 (1981).

${ }^{15}$ E. Olmedilla, L. Martínez Alonso, and F. Guil, Nuovo Cimento B 61, 49 (1981).

${ }^{16}$ See the proof of Lemma 6 of Ref. 11. Similar arguments lead to (3.8a) and (3.8b).

${ }^{17}$ E. F. Beckenbach, Bull. Amer. Math. Soc. 49, 615 (1943).

${ }^{18}$ See part $V$ of Theorem 1 of Ref. 11. The statement remains true for the matrix case.

${ }^{19}$ See Part IV of Theorem 1 of Ref. 11.

${ }^{20} \mathrm{~A}$. Degasperis, "Spectral Transform and Solvability of Nonlinear Evolution Equations" in Nonlinear Problems in Theoretical Physics, edited by A. F. Rañada, Lecture Notes in Physics, Vol. 98 (Springer, Berlin, 1979).

${ }^{21}$ A. C. Newell, "The Inverse Scattering Method" in Solitons, edited by $\mathbf{R}$ K. Bullough and P. J. Caudrey (Springer, Berlin, 1980).

${ }^{22} \mathrm{M}$. Wadati, "Generalized Matrix Form of the Inverse Scattering Method" in Solitons, edited by R. K. Bullough and P. J. Caudrey (Springer, Berlin, 1980). 